\title{
Functional Contractive Maps in Triangular Symmetric Spaces
}

\author{
Mihai Turinici \\ "A. Myller" Mathematical Seminar, Alexandru Ioan Cuza University, 700506 Iasi, Romania \\ Correspondence should be addressed to Mihai Turinici; mturi@uaic.ro \\ Received 26 November 2012; Accepted 21 January 2013 \\ Academic Editor: Ada Che \\ Copyright (C) 2013 Mihai Turinici. This is an open access article distributed under the Creative Commons Attribution License, which \\ permits unrestricted use, distribution, and reproduction in any medium, provided the original work is properly cited. \\ Some fixed point results are given for a class of functional contractions acting on (reflexive) triangular symmetric spaces. Technical \\ connections with the corresponding theories over (standard) metric and partial metric spaces are also being established.
}

\section{Introduction}

Let $X$ be a nonempty set. By a symmetric over $X$ we will mean any map $d: X \times X \rightarrow R_{+}:=[0, \infty[$ with

$$
\text { (a01) } d(x, y)=d(y, x), \forall x, y \in X \text {. }
$$

The couple $(X, d)$ will be referred to as a symmetric space. The introduction of such structures goes back to Wilson [1]; for its "modern" aspects, we refer to Hicks and Rhoades [2].

Call the symmetric $d$, triangular if

$(\mathrm{a} 02) d(x, z) \leq d(x, y)+d(y, z), \forall x, y, z \in X$,

and reflexive-triangular, provided it fulfills (the stronger condition)

$(\mathrm{a} 03) d(x, z)+d(y, y) \leq d(x, y)+d(y, z), \forall x, y, z \in X$.

The class of such particular (symmetric) spaces has multiple connections with the one of partial metric spaces, due to Matthews [3]. For, as we will see in the following, the fixed point theory for functional contractive maps in (reflexive) triangular symmetric spaces is a common root of both corresponding theories in standard metric spaces and partial metric spaces. This ultimately tells us that, for most of the function contractions taken from the list in Rhoades [4], any such theory over partial metric spaces is nothing but a clone of the corresponding one developed for standard metric spaces. Further aspects will be delineated elsewhere.

\section{Preliminaries}

Let $(X, d)$ be a symmetric space, where $d(\cdot, \cdot)$ is triangular. Call the subset $Y$ of $X, d$-singleton provided $y_{1}, y_{2} \in Y \Rightarrow$ $d\left(y_{1}, y_{2}\right)=0$.

(A) We introduce a $0 d$-convergence and a $0 d$-Cauchy structure on $X$ under the lines in Romaguera [5] (related to the context of partial metric spaces; cf. Section 4). Given the sequence $\left(x_{n}\right)$ in $X$ and the point $x \in X$, we say that $\left(x_{n}\right), 0 d$ converges to $x$ (written as $x_{n} \stackrel{0 d}{\longrightarrow} x$ ) provided $d\left(x_{n}, x\right) \rightarrow 0$ as $n \rightarrow \infty$; that is,

$$
\text { (b01) } \forall \varepsilon>0, \exists i=i(\varepsilon): n \geq i \Rightarrow d\left(x_{n}, x\right)<\varepsilon \text {. }
$$

The set of all such points $x$ will be denoted as $0 d-\lim _{n}\left(x_{n}\right)$; note that it is a $d$-singleton, because $d$ is triangular. If $0 d$ - $\lim _{n}\left(x_{n}\right)$ is nonempty, then $\left(x_{n}\right)$ is called $0 d$-convergent. We stress that the concept (b01) does not match the standard requirements in Kasahara [6], because, for the constant sequence $\left(x_{n}=u ; n \geq 0\right)$, we do not have $x_{n} \stackrel{0 d}{\rightarrow} u$ if $d(u, u)$ $\neq 0$. Further, call the sequence $\left(x_{n}\right), 0 d$-Cauchy when $d\left(x_{m}, x_{n}\right) \rightarrow 0$ as $m, n \rightarrow \infty, m \neq n$; that is,

$$
\text { (b02) } \forall \varepsilon>0, \exists j=j(\varepsilon): j \leq m<n \Rightarrow d\left(x_{m}, x_{n}\right)<\varepsilon \text {. }
$$

As $d$ is triangular, any $0 d$-convergent sequence is $0 d$-Cauchy too, but the reciprocal is not in general true. Let us say that $(X, d)$ is 0 -complete, if each $0 d$-Cauchy sequence is $0 d$ convergent.

(B) Call the sequence $\left(x_{n} ; n \geq 0\right)$, Od-semi-Cauchy provided

(b03) $d\left(x_{n}, x_{n+1}\right) \rightarrow 0$, as $n \rightarrow \infty$. 
Clearly, each $0 d$-Cauchy sequence is $0 d$-semi-Cauchy, but not conversely. The following auxiliary statement about such objects is useful for us.

Lemma 1. Let $\left(x_{n} ; n \geq 0\right)$ be a $0 d$-semi-Cauchy sequence in $X$ that is not $0 d$-Cauchy. There exist then $\varepsilon>0, j(\varepsilon) \in N$, and a couple of rank-sequences $(m(j) ; j \geq 0),(n(j) ; j \geq 0)$ with

$$
\begin{gathered}
j \leq m(j)<n(j), \quad d\left(x_{m(j)}, x_{n(j)}\right) \geq \varepsilon, \quad \forall j \geq 0, \\
n(j)-m(j) \geq 2, \quad d\left(x_{m(j)}, x_{n(j)-1}\right)<\varepsilon, \quad \forall j \geq j(\varepsilon), \\
\lim _{j} d\left(x_{m(j)+p}, x_{n(j)+q}\right)=\varepsilon, \quad \forall p, q \in\{0,1\} .
\end{gathered}
$$

Proof. As $\left(x_{n} ; n \geq 0\right)$ is not $0 d$-Cauchy, there exists, via (b02), an $\varepsilon>0$ with

$$
\begin{aligned}
& A(j):=\left\{(m, n) \in N \times N ; j \leq m<n, d\left(x_{m}, x_{n}\right) \geq \varepsilon\right\} \neq \emptyset, \\
& \forall j \geq 0 .
\end{aligned}
$$

Having this precise, denote, for each $j \geq 0$,

$$
m(j)=\operatorname{minDom}(A(j)), \quad n(j)=\min A(m(j)) .
$$

As a consequence, the couple of rank-sequences $(m(j) ; j \geq 0)$, $(n(j) ; j \geq 0)$ fulfills (1). On the other hand, letting the index $j(\varepsilon) \geq 0$ be such that

$$
d\left(x_{k}, x_{k+1}\right)<\varepsilon, \quad \forall k \geq j(\varepsilon),
$$

it is clear that (2) holds too. Finally, by the triangular property,

$$
\begin{aligned}
\varepsilon & \leq d\left(x_{m(j)}, x_{n(j)}\right) \leq d\left(x_{m(j)}, x_{n(j)-1}\right)+d\left(x_{n(j)-1}, x_{n(j)}\right) \\
& <\varepsilon+d\left(x_{n(j)-1}, x_{n(j)}\right), \quad \forall j \geq j(\varepsilon),
\end{aligned}
$$

and this establishes the case ( $p=0, q=0)$ of (3). Combining with

$$
\begin{aligned}
& d\left(x_{m(j)}, x_{n(j)}\right)-d\left(x_{n(j)}, x_{n(j)+1}\right) \\
& \quad \leq d\left(x_{m(j)}, x_{n(j)+1}\right) \\
& \quad \leq d\left(x_{m(j)}, x_{n(j)}\right)+d\left(x_{n(j)}, x_{n(j)+1}\right), \quad \forall j \geq j(\varepsilon)
\end{aligned}
$$

yields the case ( $p=0, q=1)$ of the same. The remaining situations are deductible in a similar way.

Note finally that the exposed facts do not exhaust the whole completeness theory of such objects applicable to partial metric spaces. Some complementary aspects involving these last objects may be found in Oltra and Valero [7].

(C) Let $\mathscr{F}(A)$ stand for the class of all functions from $A$ to itself. For any $\varphi \in \mathscr{F}\left(R_{+}\right)$, and any $s$ in $\left.R_{+}^{0}:=\right] 0, \infty[$, put

(b04) $L_{+} \varphi(s)=\inf _{\varepsilon>0} \Phi[s+](\varepsilon)$; where $\Phi[s+](\varepsilon)=\sup \{\varphi(t)$; $s \leq t<s+\varepsilon\}$.
By this very definition, we have the representation

$$
L_{+} \varphi(s)=\max \left\{\limsup _{t \rightarrow s+} \varphi(t), \varphi(s)\right\}, \quad \forall s \in R_{+}^{0} .
$$

Clearly, the quantity in the right member may be infinite. A basic situation when this cannot hold may be described as follows. Call $\varphi \in \mathscr{F}\left(R_{+}\right)$, normal when $[\varphi(0)=0 ; \varphi(t)<$ $t, \forall t>0]$. Note that, under such a property, one has

$$
\varphi(s) \leq L_{+} \varphi(s) \leq s, \quad \forall s \in R_{+}^{0} .
$$

The following consequence of this will be useful. Given the sequence $\left(t_{n} ; n \geq 0\right)$ in $R_{+}$and $s \in R_{+}$, define $t_{n} \downarrow s$ (as $n \rightarrow$ $\infty)$ provided $\left[t_{n} \geq s, \forall n\right]$ and $t_{n} \rightarrow s$.

Lemma 2. Let the function $\varphi \in \mathscr{F}\left(R_{+}\right)$be normal, and let $s \in R_{+}^{0}$ be arbitrarily fixed. Then,

(i) $\lim \sup _{n} \varphi\left(t_{n}\right) \leq L_{+} \varphi(s)$, for each sequence $\left(t_{n}\right)$ with $t_{n} \downarrow s$,

(ii) there exists a sequence $\left(r_{n}\right)$ with $r_{n} \downarrow s$ and $\varphi\left(r_{n}\right) \rightarrow$ $L_{+} \varphi(s)$.

Proof. (i) Given $\varepsilon>0$, there exists a rank $p(\varepsilon) \geq 0$ such that $s \leq t_{n}<s+\varepsilon$, for all $n \geq p(\varepsilon)$; hence

$$
\limsup _{n} \varphi\left(t_{n}\right) \leq \sup \left\{\varphi\left(t_{n}\right) ; n \geq p(\varepsilon)\right\} \leq \Phi[s+](\varepsilon) .
$$

It suffices taking the infimum over $\varepsilon>0$ in this relation to get the desired fact.

(ii) From (b04), $L_{+} \varphi(s)=\inf _{\varepsilon>0} \Phi[s+](\varepsilon)$, so, by the definition of infimum,

$$
\forall \varepsilon>0, \quad \exists \delta \in] 0, \varepsilon\left[: L_{+} \varphi(s) \leq \Phi[s+](\delta)<L_{+} \varphi(s)+\varepsilon .\right.
$$

This, in the case of $L_{+} \varphi(s)=0$, gives the written conclusion with $\left(r_{n}=s ; n \geq 0\right)$, for, as a direct consequence of (10), one has $\varphi(s)=0$. Suppose now that $L_{+} \varphi(s)>0$. Again from (b04),

$$
\begin{gathered}
\forall \varepsilon \in] 0, L_{+} \varphi(s)[ \\
\exists \delta \in] 0, \varepsilon\left[: L_{+} \varphi(s)-\varepsilon<L_{+} \varphi(s) \leq \Phi[s+](\delta)<L_{+} \varphi(s)+\varepsilon .\right.
\end{gathered}
$$

This, along with the definition of supremum, tells us that there must be some $r$ in $\left[s, s+\delta\left[\right.\right.$ with $L_{+} \varphi(s)-\varepsilon<\varphi(r)<L_{+} \varphi(s)+$ $\varepsilon$. Taking a sequence $\left(\varepsilon_{n}\right)$ in $R_{+}^{0}$ with $\varepsilon_{n} \rightarrow 0$, there exists a corresponding sequence $\left(r_{n}\right)$ in $R_{+}$with $r_{n} \downarrow s$ and $\varphi\left(r_{n}\right) \rightarrow$ $L_{+} \varphi(s)$, hence the conclusion.

\section{Call the normal $\varphi \in \mathscr{F}\left(R_{+}\right)$, right limit normal if}

(b05) $L_{+} \varphi(s)<s$ (or, equivalently: $\lim \sup _{t \rightarrow s+} \varphi(t)<s$ ), $\forall s \in R_{+}^{0}$.

(The last assertion is a consequence of (9) above.) In particular, the normal function $\varphi \in \mathscr{F}\left(R_{+}\right)$is right limit normal, 
whenever it is usc at the right on $R_{+}^{0}$ :

(b06) $\lim \sup _{t \rightarrow s+} \varphi(t) \leq \varphi(s)$, for each $s \in R_{+}^{0}$.

Note that this property is fulfilled when $\varphi$ is continuous at the right on $R_{+}^{0}$, for, in such a case, $\lim \sup _{t \rightarrow s+} \varphi(t)=\varphi(s)$, for all $s \in R_{+}^{0}$. Another interesting example is furnished by Lemma 2. Let us say that the normal function $\varphi \in \mathscr{F}\left(R_{+}\right)$is Geraghty normal provided (cf. Geraghty [8])

(b07) $\left(t_{n} ; n \geq 0\right) \subseteq R_{+}^{0}, \varphi\left(t_{n}\right) / t_{n} \rightarrow 1$ imply $t_{n} \rightarrow 0$.

Lemma 3. Let the normal function $\varphi \in \mathscr{F}\left(R_{+}\right)$be Geraghty normal. Then, $\varphi$ is necessarily right limit normal.

Proof. Suppose that the normal function $\varphi$ is not right limit normal. From (10), there exists some $s \in R_{+}^{0}$ with $L_{+} \varphi(s)=s$. Combining with Lemma 2 , there exists a sequence $\left(r_{n} ; n \geq 0\right)$ with $r_{n} \downarrow s$ and $\varphi\left(r_{n}\right) \rightarrow s$, whence $\varphi\left(r_{n}\right) / r_{n} \rightarrow 1$; that is, $\varphi$ is not Geraghty normal.

Remark 4. The reciprocal of this is not in general true. In fact, for the (continuous) right limit normal function $[\varphi(t)=t(1-$ $\left.\left.e^{-t}\right), t \geq 0\right]$ and the sequence $\left(t_{n}=n+1 ; n \geq 0\right)$ in $R_{+}^{0}$, we have $\varphi\left(t_{n}\right) / t_{n} \rightarrow 1$, but, evidently, $t_{n} \rightarrow \infty$.

\section{Main Result}

Let $(X, d)$ be a symmetric space, with, in addition,

(c01) $d$ is triangular and $(X, d)$ is 0 -complete.

Further, let $T: X \rightarrow X$ be a selfmap of $X$. Call $z \in X, d$-fixed if and only if $d(z, T z)=0$; the class of all such elements will be denoted as $\operatorname{Fix}(T ; d)$. Technically speaking, the points in question are obtained by a limit process as follows. Let us say that $x \in X$ is a Picard point (modulo $(d, T))$ if (i) $\left(T^{n} x ; n \geq\right.$ 0 ) is $0 d$-convergent, and (ii) each point of $0 d$ - $\lim _{n} T^{n} x$ is in $\operatorname{Fix}(T ; d)$. If this happens for each $x \in X$, then $T$ is referred to as a Picard operator (modulo $d$ ); if (in addition) $\operatorname{Fix}(T ; d)$ is $d$ singleton, then $T$ is called a global Picard operator (modulo $d$ ); compare Rus [9, Chapter 2, Section 2.2].

Now, concrete circumstances guaranteeing such properties involve (in addition to (c01)) contractive selfmaps $T$ with the $d$-asymptotic property:

(c02) $\lim _{n} d\left(T^{n} x, T^{n+1} x\right)=0, \forall x \in X$.

Precisely, denote for $x, y \in X$ :

$$
\begin{gathered}
M(x, y)=\max \{d(x, y), d(x, T x), d(y, T y)\}, \\
K(x, y)=\left(\frac{1}{2}\right)[d(x, T y)+d(T x, y)], \\
P(x, y)=\max \{M(x, y), K(x, y)\} ;
\end{gathered}
$$

and fix $G \in\{M, P\}$. Given $\varphi \in \mathscr{F}\left(R_{+}\right)$, we say that $T$ is $(d$, $G ; \varphi)$-contractive, if

(c03) $d(T x, T y) \leq \varphi(G(x, y)), \forall x, y \in X$.

The main result of this note is the following.
Theorem 5. Suppose (under (c02)) that $T$ is $(d, G ; \varphi)$ contractive, for some right limit normal function $\varphi \in \mathscr{F}\left(R_{+}\right)$. Then, $T$ is a global Picard operator (modulod).

Proof. Let $z_{1}, z_{2} \in \operatorname{Fix}(T ; d)$ be arbitrary fixed. By this very choice,

$$
M\left(z_{1}, z_{2}\right)=\max \left\{d\left(z_{1}, z_{2}\right), 0,0\right\}=d\left(z_{1}, z_{2}\right) .
$$

In addition (from the triangular property)

$$
\begin{aligned}
& d\left(z_{1}, T z_{2}\right) \leq d\left(z_{1}, z_{2}\right)+d\left(z_{2}, T z_{2}\right)=d\left(z_{1}, z_{2}\right), \\
& d\left(z_{2}, T z_{1}\right) \leq d\left(z_{2}, z_{1}\right)+d\left(z_{1}, T z_{1}\right)=d\left(z_{1}, z_{2}\right),
\end{aligned}
$$

so that $K\left(z_{1}, z_{2}\right) \leq d\left(z_{1}, z_{2}\right)$, which tells us that $P\left(z_{1}, z_{2}\right)=$ $d\left(z_{1}, z_{2}\right)$. On the other hand, again from the choice of our data and the triangular property,

$$
\begin{aligned}
d\left(z_{1}, z_{2}\right) & \leq d\left(z_{1}, T z_{1}\right)+d\left(z_{2}, T z_{2}\right)+d\left(T z_{1}, T z_{2}\right) \\
& =d\left(T z_{1}, T z_{2}\right) .
\end{aligned}
$$

Combining with the contractive condition yields (for either choice of $G$ )

$$
d\left(z_{1}, z_{2}\right) \leq \varphi\left(d\left(z_{1}, z_{2}\right)\right)
$$

wherefrom $d\left(z_{1}, z_{2}\right)=0$, so that $\operatorname{Fix}(T ; d)$ is $d$-singleton. It remains now to establish the Picard property. Fix some $x_{0} \in$ $X$, and put $x_{n}=T^{n} x_{0}, n \geq 0$; note that by (c02), $\left(x_{n} ; n \geq 0\right)$ is $0 d$-semi-Cauchy.

(I) We claim that $\left(x_{n} ; n \geq 0\right)$ is $0 d$-Cauchy. Suppose this is not true. By Lemma 1 , there exist $\varepsilon>0, j(\varepsilon) \in N$, and a couple of rank-sequences $(m(j) ; j \geq 0),(n(j) ; j \geq 0)$, with the properties (1)-(3). For simplicity, we will write (for $j \geq 0$ ), $m, n$ in place of $m(j), n(j)$, respectively. By the contractive condition,

$$
\begin{aligned}
d\left(x_{m}, x_{n}\right) \leq & d\left(x_{m}, x_{m+1}\right)+d\left(x_{n}, x_{n+1}\right) \\
& +\varphi\left(G\left(x_{m}, x_{n}\right)\right), \quad \forall j \geq j(\varepsilon) .
\end{aligned}
$$

Denote $\left(r_{j}:=M\left(x_{m}, x_{n}\right), s_{j}:=K\left(x_{m}, x_{n}\right), t_{j}:=P\left(x_{m}, x_{n}\right)\right.$; $j \geq 0$ ). From (1), $t_{j} \geq r_{j} \geq \varepsilon$, for all $j \geq j(\varepsilon)$; moreover, (3) yields $r_{j}, s_{j}, t_{j} \rightarrow \varepsilon$ as $j \rightarrow \infty$. So, passing to limit as $j \rightarrow \infty$ in (19) one gets (via Lemma 2) $\varepsilon \leq L_{+} \varphi(\varepsilon)<\varepsilon$, contradiction, so that our assertion follows.

(II) As $(X, d)$ is 0 -complete, this yields $x_{n} \stackrel{0 d}{\longrightarrow} z$ as $n \rightarrow$ $\infty$, for some $z \in X$. We claim that $z$ is an element of $\operatorname{Fix}(T ; d)$. Suppose not: that is, $\rho:=d(z, T z)>0$. By the previously mentioned properties of $\left(x_{n} ; n \geq 0\right)$, there exists $k(\rho) \in N$ such that, for all $n \geq k(\rho)$,

$$
\begin{gathered}
d\left(x_{n}, x_{n+1}\right), d\left(x_{n}, z\right)<\frac{\rho}{2}, \\
d\left(x_{n}, T z\right) \leq d\left(x_{n}, z\right)+\rho<\frac{3 \rho}{2} .
\end{gathered}
$$

This gives (again for all $n \geq k(\rho)$ )

$$
M\left(x_{n}, z\right)=\rho, \quad K\left(x_{n}, z\right)<\rho \text {; hence } P\left(x_{n}, z\right)=\rho \text {. }
$$


By the contractive condition, we then have (for either choice of $G$ )

$$
\begin{aligned}
\rho & \leq d\left(z, x_{n+1}\right)+\varphi\left(G\left(x_{n}, z\right)\right) \\
& =d\left(z, x_{n+1}\right)+\varphi(\rho), \quad \forall n \geq k(\rho) .
\end{aligned}
$$

Passing to limit as $n \rightarrow \infty$ yields $\rho \leq \varphi(\rho)$, contradiction. Hence, $z$ is an element of $\operatorname{Fix}(T ; d)$, and the proof is complete.

\section{Reflexive-Triangular Case}

Now, it remains to determine concrete circumstances under which $T$ is $d$-asymptotic. Let $(X, d)$ be a symmetric space, with

(d01) $d$ is reflexive-triangular and $(X, d)$ is 0 -complete.

Further, let $T$ be a selfmap of $X$; and fix $G \in\{M, P\}$.

Lemma 6. Suppose that $T$ is $(d, G ; \varphi)$-contractive, for some right limit normal function $\varphi \in \mathscr{F}\left(R_{+}\right)$. Then, $T$ is $d$ asymptotic.

Proof. By definition, we have

$$
M(x, T x)=\max \left\{d(x, T x), d\left(T x, T^{2} x\right)\right\}, \quad \forall x \in X
$$

On the other hand, by the reflexive-triangular property,

$$
\begin{aligned}
K(x, T x) & \leq\left(\frac{1}{2}\right)\left[d(x, T x)+d\left(T x, T^{2} x\right)\right] \\
& \leq \max \left\{d(x, T x), d\left(T x, T^{2} x\right)\right\} .
\end{aligned}
$$

So, by simply combining these,

$$
P(x, T x)=\max \left\{d(x, T x), d\left(T x, T^{2} x\right)\right\}, \quad \forall x \in X .
$$

Fix some $x \in X$, and put $\left(\rho_{n}:=d\left(T^{n} x, T^{n+1} x\right) ; n \geq 0\right)$. From the contractive condition, $\left[\rho_{n+1} \leq \varphi\left(\max \left\{\rho_{n}, \rho_{n+1}\right\}\right), \forall n \geq 0\right]$. As $\varphi$ is normal, this yields

$$
\rho_{n+1} \leq \varphi\left(\rho_{n}\right), \quad \forall n \geq 0 .
$$

In particular, $\left(\rho_{n} ; n \geq 0\right)$ is descending; hence, $\rho:=\lim _{n} \rho_{n}$ exists in $R_{+}$. Assume that $\rho>0$. By Lemma 2, we must have $\rho \leq L_{+} \varphi(\rho)<\rho$; contradiction. Hence, $\rho=0$, and the conclusion follows.

Now, by simply combining this with Theorem 5 , we have (under (d01)) the following.

Theorem 7. Suppose that $T$ is $(d, G ; \varphi)$-contractive, for some right limit normal function $\varphi \in \mathscr{F}\left(R_{+}\right)$. Then, $T$ is a global Picard operator (modulod).

A basic particular case of this result is the following. Call the symmetric $d$ on $X$ an almost partial metric provided it is reflexive-triangular and $(\mathrm{d} 02) d(x, y)=0 \Rightarrow x=y(d$ is sufficient $)$.

Note that, in such a case,

$\left[\forall Y \in \mathscr{P}_{0}(X)\right] ; \quad Y$ is $d$-singleton $\Longrightarrow Y$ is singleton,

$$
\operatorname{Fix}(T ; d) \subseteq \operatorname{Fix}(T)
$$

(=the class of all fixed points of $T$ in $X$ ).

Assume in the following that

$(\mathrm{d} 03) d$ is almost partial metric and $(X, d)$ is 0 -complete.

Theorem 8. Let the selfmap $T$ be $(d, G ; \varphi)$-contractive, for some right limit normal function $\varphi \in \mathscr{F}\left(R_{+}\right)$. Then,

$$
\begin{gathered}
\operatorname{Fix}(T ; d)=\operatorname{Fix}(T)=\{z\}, \quad \text { where } d(z, z)=0, \\
T^{n} x \stackrel{0 d}{\longrightarrow} z \text { as } n \longrightarrow \infty, \forall x \in X .
\end{gathered}
$$

Proof. By Theorem 7, we have (taking (27)+(28) into account)

$$
\operatorname{Fix}(T ; d)=\{z\}, \quad \text { with } z \in \operatorname{Fix}(T), d(z, z)=0,
$$

and, moreover, (30) holds. It remains to establish that $\operatorname{Fix}(T)=\{z\}$. For each $w \in \operatorname{Fix}(T)$, we must have (by (30)) $T^{n} w \stackrel{0 d}{\longrightarrow} z$, which means $d(w, z)=0$; hence (as $d=$ sufficient) $w=z$. The proof is complete.

Now, let us give two important examples of such objects.

(A) Clearly, each (standard) metric on $X$ is an almost partial metric. Then, Theorem 8 is just the main result in Jachymski [10]; see also Cho et al. [11]. In fact, its argument mimics the one in those papers. The only "specific" fact to be underlined is related to the reflexive-triangular property of our symmetric $d$.

(B) According to Matthews [3], call the symmetric $d$, a partial metric provided it is reflexive-triangular and

$(\mathrm{d} 04)[d(x, x)=d(y, y)=d(x, y)] \Rightarrow x=y(d$ is strongly sufficient)

(d05) $\max \{d(x, x), d(y, y)\} \leq d(x, y), \forall x, y \in X$ (Matthews property).

Note that, by the reflexive-triangular property, one has (with $z=x$ )

$$
d(x, x)+d(y, y) \leq 2 d(x, y), \quad \forall x, y \in X,
$$

and this, along with (d04), yields (d02); that is, each partial metric is an almost partial metric. Hence, Theorem 8 is applicable to such objects; its corresponding form is just the main result in Romaguera [12]; see also Altun et al. [13]. It is to be stressed here that the Matthews property (d05) was not used in the quoted statement. This forces us to conclude that this property is not effective in most fixed point results based on such contractive conditions. On the other hand, the argument used here is, practically, a clone of that developed for the standard metric setting. Hence-at 
least for such results - it cannot get us new insights for the considered matter. Clearly, the introduction of an additional order structure on $X$ does not change this conclusion. Hence, the results in the area due to Altun and Erduran [14] are but formal copies of the ones (in standard metric spaces) due to Agarwal et al. [15]. This is also true for the common fixed points question, when, for example, the results in Shobkolaei et al. [16] or Karapınar and Shatanawi [17] are but a translation of the ones (in standard metric spaces) due to Jachymski [18]. Finally, we may ask whether this reduction scheme comprises as well the class of contraction maps in general complete partial metric spaces taken as in Ilić et al. [19]. Formally, such results are not reducible to the previous ones. But, from a technical perspective, this is possible; see Turinici [20] for details.

\section{Triangular Symmetrics}

Let $(X, d)$ be a symmetric space, taken as in (c01), and let $T$ be a selfmap of $X$.

Lemma 9. Suppose that $T$ is $(d, M ; \varphi)$-contractive, for some right limit normal function $\varphi \in \mathscr{F}\left(R_{+}\right)$. Then, $T$ is $d$ asymptotic.

The argument is based on the evaluation (23); see also Zhu et al. [21].

Now, by simply combining this with Theorem 5, we have (under (c01)) the following.

Theorem 10. Suppose that $T$ is $(d, M ; \varphi)$-contractive, for some right limit normal function $\varphi \in \mathscr{F}\left(R_{+}\right)$. Then, $T$ is a global Picard operator (modulod).

A basic particular case of this result is to be stated as below. Call the symmetric $d(\cdot, \cdot)$, a weak almost partial metric provided it is triangular and sufficient (i.e., (d02) holds). Note that, in such a case, relations (27) and (28) are still retainable. Assume in the following that

(e01) $d$ is a weak almost partial metric and $(X, d)$ is 0 complete.

Theorem 11. Let the selfmap $T$ be $(d, M ; \varphi)$-contractive, for some right limit normal function $\varphi \in \mathscr{F}\left(R_{+}\right)$. Then, conclusions of Theorem 8 are holding.

The proof mimics the one of Theorem 8 (if one takes Theorem 10 as starting point), so, it will be omitted.

Now, let us give two important examples of such objects.

(A) Clearly, each (standard) metric on $X$ is a weak almost partial metric. Then, Theorem 11 is comparable with the main result in Jachymski [10].

(B) Remember that the symmetric $d$ is called a partial metric provided it is reflexive-triangular and $(\mathrm{d} 04)+(\mathrm{d} 05)$ hold. As before, (32) tells us (via (d04)) that each partial metric is a weak almost partial metric; hence, Theorem 11 is applicable to such objects. In particular, when $\varphi$ is linear $\left(\varphi(t)=\lambda t, t \in R_{+}\right.$, for some $\lambda \in[0,1[)$, one recovers the Banach type fixed point result in Aage and Salunke [22], which, in turn, includes the one in Valero [23]. On the other hand, Lemma 3 tells us that Theorem 11 includes as well a related fixed point statement due to Dukić et al. [24]; see also Golubović et al. [25]; moreover, by Remark 4, the converse inclusion is not in general true. It is to be stressed here that the Matthews property (d05) was not effectively used in the quoted statement; in addition, the (stronger) reflexivetriangular property of $d$ was replaced by the triangular property of the same. As before, the argument used here is, practically, a clone of that developed in the standard metric setting; whence, the results we just quoted are technically deductible from the one in Boyd and Wong [26]. Further developments of these results may be performed under the lines in Turinici [27].

\section{Acknowledgment}

The author is very indebted to both referees, for a number of useful suggestions.

\section{References}

[1] W. A. Wilson, "On Semi-Metric Spaces," The American Journal of Mathematics, vol. 53, no. 2, pp. 361-373, 1931.

[2] T. L. Hicks and B. E. Rhoades, "Fixed point theory in symmetric spaces with applications to probabilistic spaces," Nonlinear Analysis A, vol. 36, no. 3, pp. 331-344, 1999.

[3] S. G. Matthews, "Partial metric topology", in Papers on General Topology and Applications, vol. 728, pp. 183-197, Academy of Sciences, New York, NY, USA, 1994.

[4] B. E. Rhoades, "A comparison of various definitions of contractive mappings," Transactions of the American Mathematical Society, vol. 226, pp. 257-290, 1977.

[5] S. Romaguera, "A Kirk type characterization of completeness for partial metric spaces," Fixed Point Theory and Applications, vol. 2010, Article ID 493298, 6 pages, 2010.

[6] S. Kasahara, "On some generalizations of the Banach contraction theorem," Kyoto University. Research Institute for Mathematical Sciences. Publications, vol. 12, no. 2, pp. 427-437, 1976.

[7] S. Oltra and O. Valero, "Banach's fixed point theorem for partial metric spaces," Rendiconti dell'Istituto di Matematica dell'Università di Trieste, vol. 36, no. 1-2, pp. 17-26, 2005.

[8] M. A. Geraghty, "On contractive mappings," Proceedings of the American Mathematical Society, vol. 40, pp. 604-608, 1973.

[9] I. A. Rus, Generalized Contractions and Applications, Cluj University Press, Cluj, Romania, 2001.

[10] J. Jachymski, "A generalization of the theorem by Rhoades and Watson for contractive type mappings," Mathematica Japonica, vol. 38, no. 6, pp. 1095-1102, 1993.

[11] Y. J. Cho, B. K. Sharma, and D. R. Sahu, "Semi-compatibility and fixed points," Mathematica Japonica, vol. 42, no. 1, pp. 9198, 1995.

[12] S. Romaguera, "Fixed point theorems for generalized contractions on partial metric spaces," Topology and its Applications, vol. 159, no. 1, pp. 194-199, 2012.

[13] I. Altun, F. Sola, and H. Simsek, "Generalized contractions on partial metric spaces," Topology and its Applications, vol. 157, no. 18, pp. 2778-2785, 2010. 
[14] I. Altun and A. Erduran, "Fixed point theorems for monotone mappings on partial metric spaces," Fixed Point Theory and Applications, vol. 2011, Article ID 508730, 10 pages, 2011.

[15] R. P. Agarwal, M. A. El-Gebeily, and D. O’Regan, "Generalized contractions in partially ordered metric spaces," Applicable Analysis, vol. 87, no. 1, pp. 109-116, 2008.

[16] N. Shobkolaei, S. M. Vaezpour, and S. Sedghi, "A common fixed point theorem on ordered partial metric spaces," Journal of Basic and Applied Scientific Research, vol. 1, pp. 3433-3439, 2011.

[17] E. Karapınar and W. Shatanawi, "On weakly $(C, \psi, \varphi)$ contractive mappings in ordered partial metric spaces," Abstract and Applied Analysis, vol. 2012, Article ID 495892, 17 pages, 2012.

[18] J. Jachymski, "Common fixed point theorems for some families of maps," Indian Journal of Pure and Applied Mathematics, vol. 25, no. 9, pp. 925-937, 1994.

[19] D. Ilić, V. Pavlović, and V. Rakočević, "Extensions of the Zamfirescu theorem to partial metric spaces," Mathematical and Computer Modelling, vol. 55, no. 3-4, pp. 801-809, 2012.

[20] M. Turinici, "Function contractive maps in partial metric spaces," Romai, vol. 8, pp. 189-207, 2012.

[21] J. Zhu, Y. J. Cho, and S. M. Kang, "Equivalent contractive conditions in symmetric spaces," Computers \& Mathematics with Applications, vol. 50, no. 10-12, pp. 1621-1628, 2005.

[22] C. T. Aage and J. N. Salunke, "Some results of fixed point theorem in dislocated quasi-metric spaces," Bulletin Marathwada Mathematical Society, vol. 9, pp. 1-5, 2008.

[23] O. Valero, "On Banach fixed point theorems for partial metric spaces," Applied General Topology, vol. 6, no. 2, pp. 229-240, 2005.

[24] D. Dukić, Z. Kadelburg, and S. Radenović, "Fixed points of Geraghty-type mappings in various generalized metric spaces," Abstract and Applied Analysis, vol. 2011, Article ID 561245, 13 pages, 2011.

[25] Z. Golubović, Z. Kadelburg, and S. Radenović, "Coupled coincidence points of mappings in ordered partial metric spaces," Abstract and Applied Analysis, vol. 2012, Article ID 192581, 18 pages, 2012.

[26] D. W. Boyd and J. S. W. Wong, "On nonlinear contractions," Proceedings of the American Mathematical Society, vol. 20, pp. 458-464, 1969.

[27] M. Turinici, "Abstract comparison principles and multivariable Gronwall-Bellman inequalities," Journal of Mathematical Analysis and Applications, vol. 117, no. 1, pp. 100-127, 1986. 


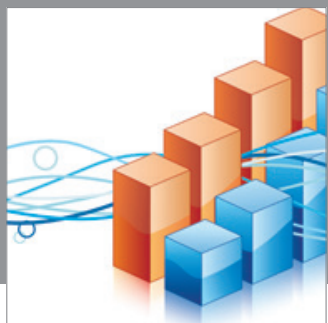

Advances in

Operations Research

mansans

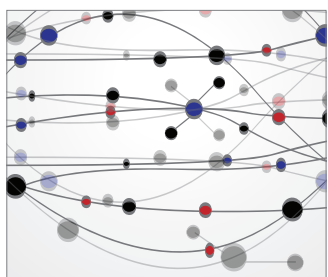

The Scientific World Journal
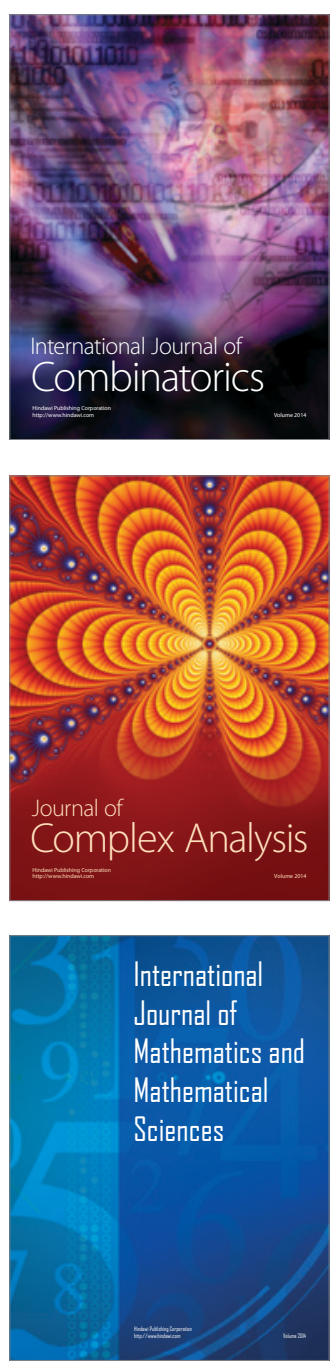
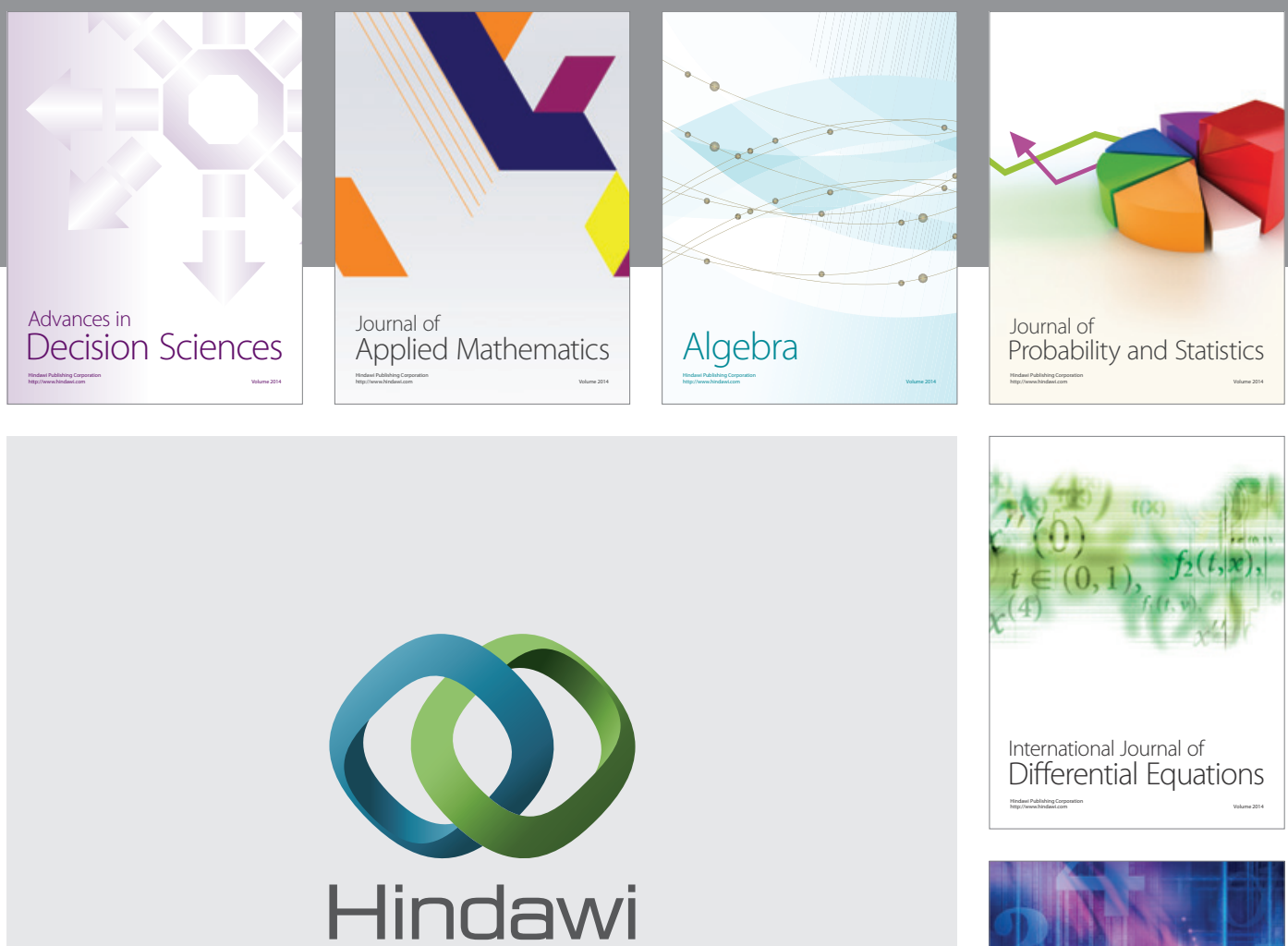

Submit your manuscripts at http://www.hindawi.com
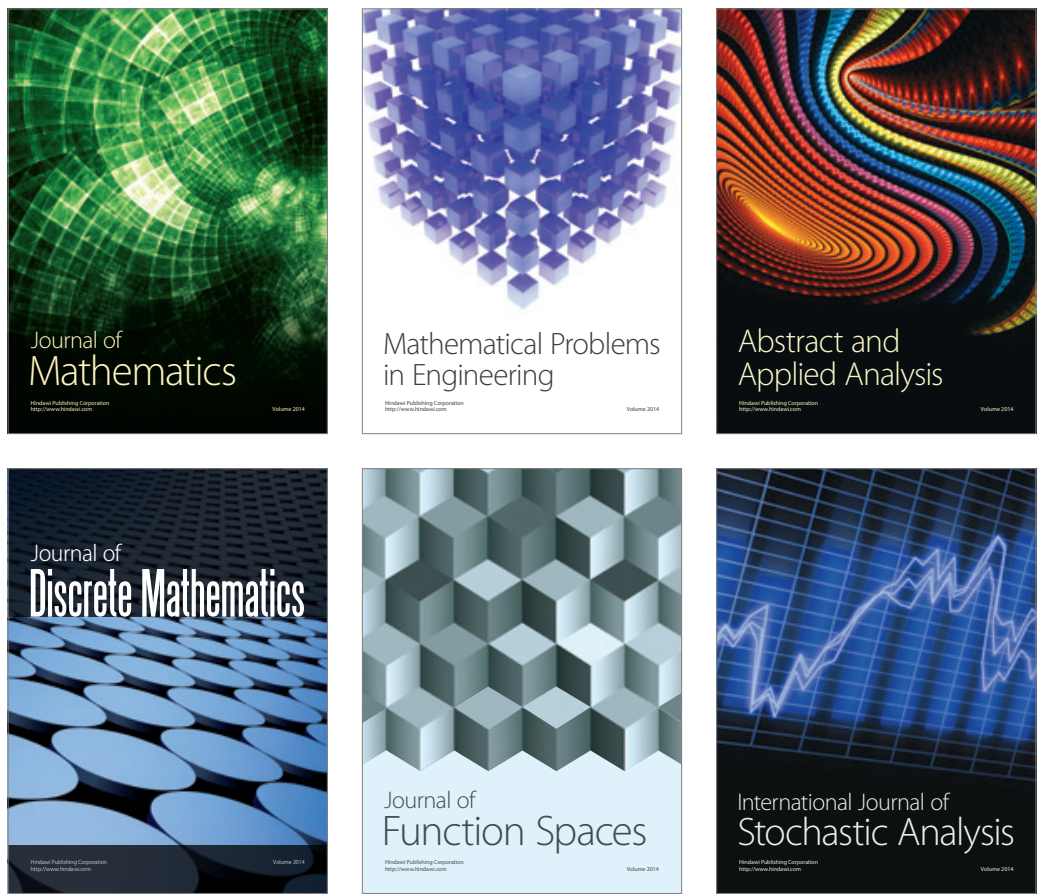

Journal of

Function Spaces

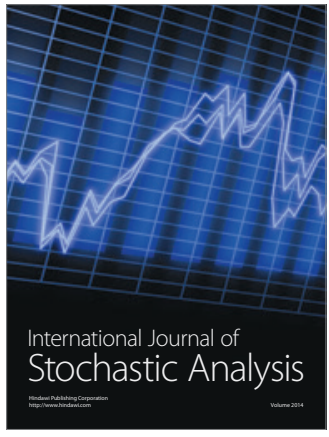

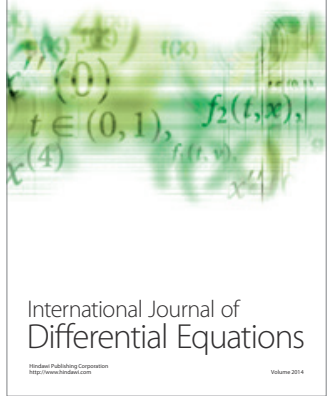
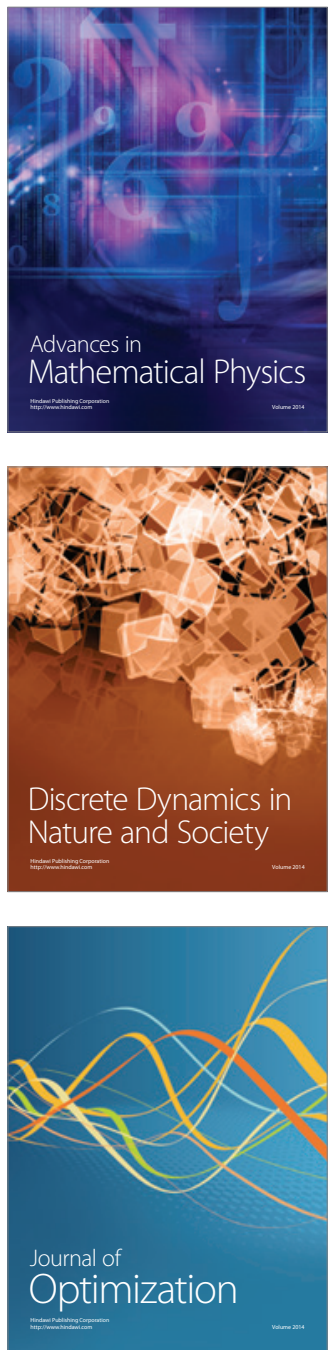